\title{
A Survey on Localization for Mobile Wireless Sensor Networks
}

\author{
Isaac Amundson and Xenofon D. Koutsoukos \\ Institute for Software Integrated Systems (ISIS) \\ Department of Electrical Engineering and Computer Science \\ Vanderbilt University \\ Nashville, TN 37235, USA \\ isaac.amundson@vanderbilt.edu
}

\begin{abstract}
Over the past decade we have witnessed the evolution of wireless sensor networks, with advancements in hardware design, communication protocols, resource efficiency, and other aspects. Recently, there has been much focus on mobile sensor networks, and we have even seen the development of small-profile sensing devices that are able to control their own movement. Although it has been shown that mobility alleviates several issues relating to sensor network coverage and connectivity, many challenges remain. Among these, the need for position estimation is perhaps the most important. Not only is localization required to understand sensor data in a spatial context, but also for navigation, a key feature of mobile sensors. In this paper, we present a survey on localization methods for mobile wireless sensor networks. We provide taxonomies for mobile wireless sensors and localization, including common architectures, measurement techniques, and localization algorithms. We conclude with a description of real-world mobile sensor applications that require position estimation.
\end{abstract}

\section{Introduction}

Wireless sensor network (WSN) applications typically involve the observation of some physical phenomenon through sampling of the environment. Mobile wireless sensor networks (MWSNs) are a particular class of WSN in which mobility plays a key role in the execution of the application. In recent years, mobility has become an important area of research for the WSN community. Although WSN deployments were never envisioned to be fully static, mobility was initially regarded as having several challenges that needed to be overcome, including connectivity, coverage, and energy consumption, among others. However, recent studies have been showing mobility in a more favorable light 1. Rather than complicating these issues, it has been demonstrated that the introduction of mobile entities can resolve some of these problems [2. In addition, mobility enables sensor nodes to target and track moving phenomena such as chemical clouds, vehicles, and packages [3].

One of the most significant challenges for MWSNs is the need for localization. In order to understand sensor data in a spatial context, or for proper navigation

R. Fuller and X.D. Koutsoukos (Eds.): MELT 2009, LNCS 5801, pp. 235254 2009.

(C) Springer-Verlag Berlin Heidelberg 2009 
throughout a sensing region, sensor position must be known. Because sensor nodes may be deployed dynamically (i.e., dropped from an aircraft), or may change position during run-time (i.e., when attached to a shipping container), there may be no way of knowing the location of each node at any given time. For static WSNs, this is not as much of a problem because once node positions have been determined, they are unlikely to change. On the other hand, mobile sensors must frequently estimate their position, which takes time and energy, and consumes other resources needed by the sensing application. Furthermore, localization schemes that provide high-accuracy positioning information in WSNs cannot be employed by mobile sensors, because they typically require centralized processing, take too long to run, or make assumptions about the environment or network topology that do not apply to dynamic networks.

This paper presents a survey and taxonomy of localization methods for mobile wireless sensor networks. Localization is a well-studied problem in several areas including robotics, mobile ad hoc and vehicular networks, and wireless sensor networks. Here, we focus solely on those methodologies that relate directly to MWSNs. In order to understand localization in the context of mobility, we begin with an overview on mobile wireless sensor networks. The overview includes common MWSN architectures, discusses the advantages of adding mobility, and describes differences with WSNs. We then provide a taxonomy of localization methods in MWSNs. In addition, we discuss the impact centralized processing and the environment have on MWSN localization. Finally, we describe real-world MWSN applications that require position estimation.

At present, the most widely used method for localization is the NAVSTAR Global Positioning System (GPS) 4. The system consists of approximately 24 satellites that orbit the planet, of which four are required to obtain location information (3 to determine 3D position, and 1 to resolve local clock uncertainty). The satellites continuously transmit messages that contain ephemeris data, transmission time, and vital statistics. Mobile receivers are then able to compute their location using lateration based on signal time of flight and orbital position data. Commercial-use GPS is accurate to within 10 meters, is free to use anywhere on the planet and, for many mobile applications, is an ideal localization technology that should be taken advantage of. However, there are also several situations in which it will not work reliably. Because GPS requires line of sight to multiple satellites, mobile sensor networks that are deployed in urban environments, indoors, underground, or off-planet will not be able to use it. Furthermore, although GPS receivers are available for mote-scale devices, they are still relatively expensive, and therefore undesirable for many deployments. Therefore, in this survey, localization is presented from a GPS-less perspective (i.e., one that does not rely solely on GPS technology).

The survey is organized as follows. In Section 2, we provide a description of the key features of mobile wireless sensor networks. Section 3 then focuses specifically on localization in MWSNs, and includes a taxonomy of localization methods. Section 4 continues with a description of MWSN applications that require localization. Section 5 concludes. 


\section{Mobile Wireless Sensor Networks}

In this section, we provide a brief taxonomy of MWSNs, including the differences between MWSNs and WSNs, and the advantages of adding mobility.

\section{$2.1 \quad$ MWSN Architectures}

Mobile sensor networks can be categorized by flat, 2-tier, or 3-tier hierarchical architectures [5], as illustrated in Figure 1, and described below.

(a)

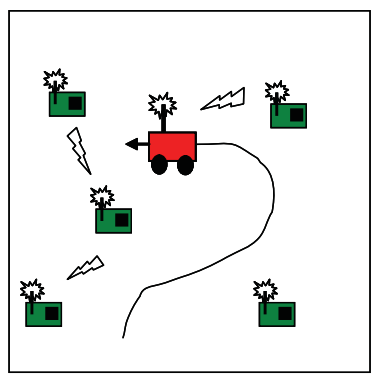

Fig. 1. (a) Flat, (b) 2-Tier, and (c) 3-Tier MWSN architectures (c)
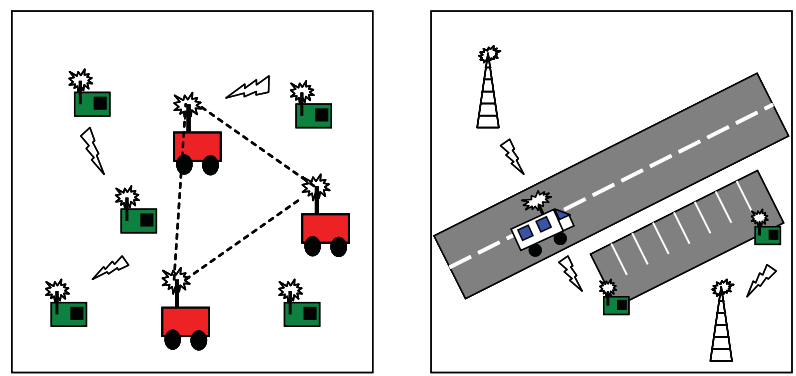

Alat, or planar, network architecture comprises a set of heterogeneous devices that communicate in an ad hoc manner. The devices can be mobile or stationary, but all communicate over the same network. Basic navigation systems such as 6 . have a flat architecture, as pictured in Figure 1 .

The two-tier architecture consists of a set of stationary nodes, and a set of mobile nodes. The mobile nodes form an overlay network or act as data mules to help move data through the network. The overlay network can include mobile devices that have greater processing capability, longer communication range, and higher bandwidth. Furthermore, the overlay network density may be such that all nodes are always connected, or the network can become disjoint. When the latter is the case, mobile entities can position themselves in order to re-establish connectivity, ensuring network packets reach their intended destination. The NavMote system [7] takes this approach. The 2-tier architecture is pictured in Figure 10.

In the three-tier architecture, a set of stationary sensor nodes pass data to a set of mobile devices, which then forward that data to a set of access points. This heterogeneous network is designed to cover wide areas and be compatible with several applications simultaneously. For example, consider a sensor network application that monitors a parking garage for parking space availability. The sensor network (first tier) broadcasts availability updates to compatible mobile devices (second tier), such as cell phones or PDAs, that are passing by. In turn, 
the cell phones forward this availability data to access points (third tier), such as cell towers, and the data are uploaded into a centralized database server. Users wishing to locate an available parking space can then access the database. The 3 -tier architecture is pictured in Figure 1.

At the node level, mobile wireless sensors can be categorized based on their role within the network:

Mobile Embedded Sensor. Mobile embedded nodes do not control their own movement; rather, their motion is directed by some external force, such as when tethered to an animal [8] or attached to a shipping container [9]. Typical embedded sensors include [10], 11, and [12.

Mobile Actuated Sensor. Sensor nodes can also have locomotion capability (for example, [13, [14, [15]), which enables them to move throughout a sensing region [6]. With this type of controlled mobility, the deployment specification can be more exact, coverage can be maximized, and specific phenomena can be targeted and followed.

Data Mule. Oftentimes, the sensors need not be mobile, but they may require a mobile device to collect their data and deliver it to a base station. These types of mobile entities are referred to as data mules [16. It is generally assumed that data mules can recharge their power source automatically.

Access Point. In sparse networks, or when a node drops off the network, mobile nodes can position themselves to maintain network connectivity [16], [17. In this case, they behave as network access points.

\subsection{Advantages of Adding Mobility}

Sensor network deployments are often determined by the application. Nodes can be placed in a grid, randomly, surrounding an object of interest, or in countless other arrangements. In many situations, an optimal deployment is unknown until the sensor nodes start collecting and processing data. For deployments in remote or wide areas, rearranging node positions is generally infeasible. However, when nodes are mobile, redeployment is possible. In fact, it has been shown [17, [18] that the integration of mobile entities into WSNs improves coverage, and hence, utility of the sensor network deployment. This enables more versatile sensing applications as well [1]. For example, Figure 2]illustrates a mobile sensor network that monitors wildfires. The mobile sensors are able to maintain a safe distance from the fire perimeter, as well as provide updates to fire fighters that indicate where that perimeter currently is.

In networks that are sparse or disjoint, or when stationary nodes die, mobile nodes can maneuver to connect the lost or weak communication pathways. This is not possible with static WSNs, in which the data from dead or disconnected nodes would simply be lost. Similarly, when network sinks are stationary, nodes closer to the base station will die sooner, because they must forward more data messages than those nodes further away. By using mobile base stations, this problem is eliminated, and the lifetime of the network is extended [19]. 


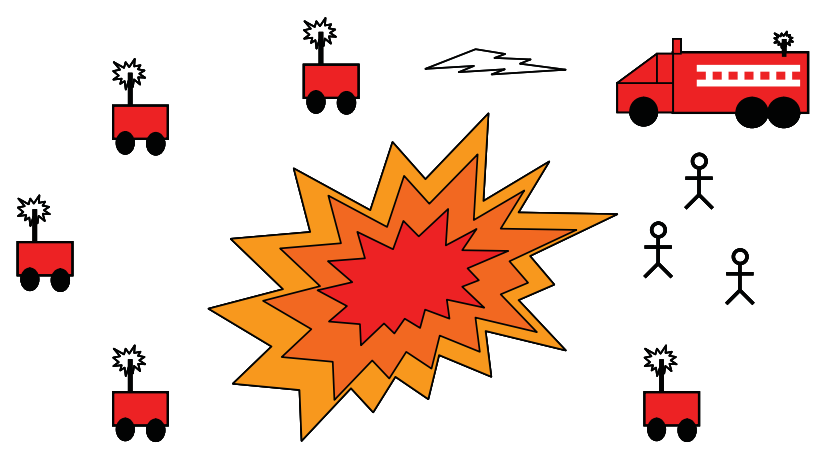

Fig. 2. A MWSN that monitors wildfires. As the fire spreads, the mobile sensors can track it, as well as stay out of its way.

Mobility also enables greater channel capacity and maintains data integrity by creating multiple communication pathways, and reducing the number of hops messages must travel before reaching their destination [20].

\subsection{Differences between WSNs and MWSNs}

In order to focus on the mobility aspect of wireless sensor networks, it is important to first understand how the common assumptions regarding staticallydeployed WSNs change when mobile entities are introduced.

Localization. In statically deployed networks, node position can be determined once during initialization. However, those nodes that are mobile must continuously obtain their position as they traverse the sensing region. This requires additional time and energy, as well as the availability of a rapid localization service.

Dynamic Network Topology. Traditional WSN routing protocols 21, which describe how to pass messages through the network so they will most likely reach their destination, typically rely on routing tables or recent route histories. In dynamic topologies, table data become outdated quickly, and route discovery must repeatedly be performed at a substantial cost in terms of power, time, and bandwidth. Fortunately, there is an active area of research dedicated to routing in mobile ad hoc networks (MANETs), and MWSNs can borrow from this work [22].

Power Consumption. Power consumption models [23] differ greatly between WSNs and MWSNs. For both types of networks, wireless communication incurs a significant energy cost and must be used efficiently. However, mobile entities require additional power for mobility, and are often equipped with a much larger energy reserve, or have self-charging capability that enables them to plug into the power grid to recharge their batteries.

Network Sink. In centralized WSN applications, sensor data is forwarded to a base station, where it can be processed using resource-intensive methods. Data routing and aggregation can incur significant overhead. Some MWSNs use mobile base stations [19, which traverse the sensing region to collect data, or 
(a)

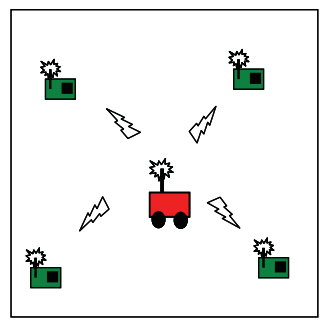

Fig. 3. Localization phases estimation (b)

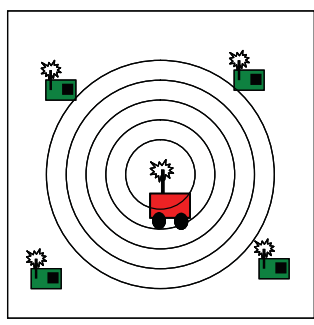

(c)

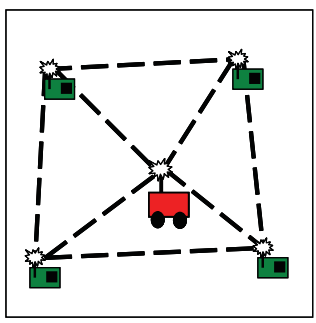

(a) coordination, (b) measurement, and (c) position

position themselves so that the number of transmission hops is minimized for the sensor nodes.

\section{Localization in MWSNs}

In this section, we provide a taxonomy of localization methods for MWSNs, as well as survey selected works representative of common MWSN localization. An extensive library of WSN localization research has been published within the past decade 24], 25, and many of these techniques can be applied to MWSNs. The localization techniques use diverse hardware, algorithms and signal modalities, which can be categorized along several different dimensions. We start by describing the three phases typically used in localization ([26, [27, [28]): (1) coordination, (2) measurement, and (3) position estimation. We then focus on other aspects of MWSN-based localization, such as the effects of mobility, centralized versus distributed processing, and the environment.

MWSN localization is typically performed as illustrated in Figure 3 A group of nodes coordinate to initiate localization. One or more nodes then emit a signal, and some property of the signal (e.g. arrival time, phase, signal strength, etc.) is observed by one or more receivers. Node position is then determined by transforming signal measurements into position estimates by means of a localization algorithm. In order to determine position, it is often necessary to enlist the help of cooperating sensor nodes that have been deployed into the environment at known positions a priori. These devices are referred to as anchor, infrastructure, or seed nodes. For example, in GPS, the infrastructure nodes are the satellites that orbit the planet. The position estimate may be relative to a set of stationary anchor nodes at known positions in a local coordinate system, or absolute coordinates may be obtained if the positions of the anchor nodes are known with respect to some global coordinate system (i.e., using GPS).

\subsection{Coordination Phase}

Prior to signal transmission, nodes participating in the localization typically coordinate with one another. Such coordination can include notification that the 
localization process is about to begin, and clock synchronization, which enables received signal data to be analyzed within a common timeframe. Coordination techniques such as reference broadcast synchronization (RBS) [29] and elapsed time on arrival (ETA) 30] exist that encapsulate both notification and synchronization into a single message. These coordination methods have microsecond accuracy and require transmission of only a single message. For example, the SyncEvent, one of the ETA primitives, declares a time in the future to begin the localization process. Encoded in the message is the timestamp of the message sender (typically the localization coordinator), which is inserted into the message immediately before transmission, thus reducing the amount of non-deterministic latency involved in the synchronization. All nodes within broadcast range will receive the message at approximately the same time instant, and assuming a negligible transit time of the radio signal through air, will be able to transform the sender timestamp into their local timescale. This technique is used in several localization schemes, including [31, 32, 9], and 6].

\subsection{Measurement Phase}

The measurement phase typically involves the transmission of a signal by at least one node, followed by signal processing on the other participating nodes.

Signal Modalities. The choice of signal modality used by sensor nodes is important for accurate localization, and depends on node hardware, the environment, and the application. Because WSNs are developed to provide inexpensive wide-area observation capability, it is generally undesirable to add additional hardware to the sensor board, because this increases cost and power consumption. Localization schemes will also perform differently in different environments. In humid environments, for example, radio signals perform worse than acoustic signals because moisture in the air absorbs and reflects the high frequency radio waves but does little to affect the vibrational sound waves. Finally, the application itself places some constraints on signal modality. A military application, for example, in which nodes must localize under stealth conditions, would be much better off using a silent modality such as radio frequency, rather than an audible one such as acoustic.

The acoustic modality typically employs either ultrasound or audible wave propagation. Several techniques have been published for each. Two early and commonly cited ultrasound localization techniques are Active Bats [33 and Cricket 34]. A more recent ultrasound approach, which includes a survey on ultrasonic positioning systems and challenges can be found in [35. In the audible acoustic band, several novel localization systems have been developed, including beamforming [36], a sniper detection system [37, and generalized sound source localization [38].

Infrared (IR) signal attenuation is relatively high, requiring close proximity between transmitter and receiver. This is acceptable for most indoor localization schemes, however, outdoor localization becomes difficult, not only due to proximity issues, but also because the IR signal is difficult to read in the presence 


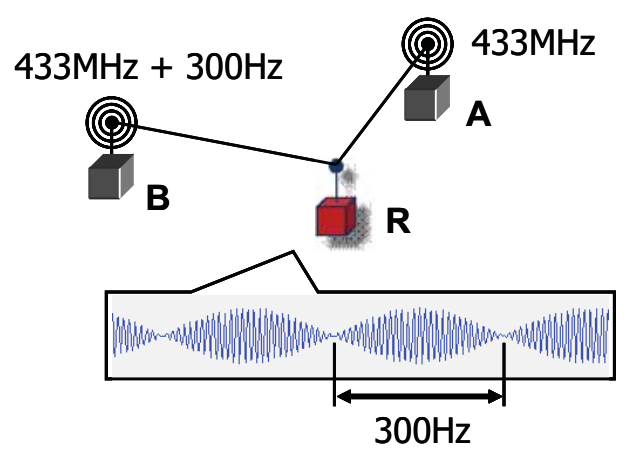

Fig. 4. Radio interferometry. Two nodes transmit a sinusoidal signal at slightly different frequencies, which interfere to create a low-frequency beat signal that can be measured using resource-constrained sensor nodes.

of sunlight. One of the earliest mobile localization systems is the Active Badge system [39], whereby a small electronic device (badge), carried by a user, emits a periodic identification signal. The signal is received by infrastructure nodes and centrally processed, allowing position information to be accessed by authorized users. Other IR localization methods can be found in [40] and 41.

Because all wireless sensor nodes have onboard radio hardware, radio frequency $(\mathrm{RF})$ propagation has become a popular signal modality for localization. Signal properties such as strength, phase, or frequency are analyzed to derive range data for position estimation. One benefit of using $R F$ is that it has been shown to achieve localization accuracy on the order of centimeters, even in sparse networks [42]. On the other hand, because typical sensor node radios transmit at frequencies between $400 \mathrm{MHz}$ to $2.6 \mathrm{GHz}$, sampling the raw signal for phase or frequency cannot be done with resource-constrained hardware. Instead, methods such as radio interferometry [31] must be used to generate a low frequency beat signal, as shown in Figure 4 . The frequency and phase of the beat signal can then be measured by observing the received signal strength indicator on the radio chip.

The Lighthouse 43 and Spotlight 44 localization techniques use a light beacon to determine node position. Although both methods claim high accuracy, they require line of sight, a powerful light source that will perform well in lighted areas, and customized hardware for the light source.

Measurement Techniques. Several techniques exist for obtaining bearing, range, or proximity information based on signal measurement.

The angle-of-arrival (AOA) method [45, 46], 47], 36] involves determining the angular separation between two beacons, or a single beacon and a fixed axis. By determining the AOA at a certain number of sensor nodes, position can be determined by angulation methods, as outlined in Section 3.3 .

Localization by time-of-arrival (TOA) [48, [49, [50] measures the time a signal takes to arrive at some number of sensors. This requires knowing the time the signal was transmitted, and assumes tight time synchronization between 
sender and receiver. The signal will have known propagation properties, such as speed through air at sea level. The main drawback of this approach is that it is difficult to precisely record the arrival time of radio signals, since they travel close to the speed of light. Therefore, it works best with an acoustic source. In addition, after transmitting the signal, the source must also make its transmit time known, incurring additional communication overhead. This can be avoided by employing a round-trip TOA method [51, whereby Node A transmits a signal to Node B. Upon signal reception, Node B transmits a signal back, and Node A observes the round-trip time, accounting for deterministic delay during the communication process.

Time-difference-of-arrival (TDOA) localization [32, 38, improves upon the TOA approach by eliminating the need to know when the signal was transmitted. Several time-synchronized nodes receive a signal, and look at the difference in arrival times (or difference in signal phase) at a specific time instant. Because the signal travels at a constant speed, the source position can easily be determined if there are a sufficient number of participating nodes.

Another localization method examines the received signal strength (RSS) of a message broadcast from a known location [52, [53. Since the free-space signal strength model is governed by the inverse-square law, accurate localization is possible. Furthermore, this typically does not involve any hardware modifications because most chips (e.g. RF, IR, etc.) provide software access to the amplitude of the received signal. Another use for RSS is profiling 54, 55, in which a map of RSS values is constructed during an initial training phase. Sensors then estimate their position by matching observed RSS values with the training data.

Recently, there have been several published techniques that determine the position of a node based on the observed frequency of a signal [9], 6], [56], [57]. Signal frequency will undergo Doppler-shift when the transmitter and receiver are moving relative to one another. The observed Doppler-shift at multiple infrastructure nodes can be used to derive the position and velocity of the mobile node.

The above techniques provide the most accurate position estimates, however, it is oftentimes sufficient to only localize to a region. Such a region might be a room in a house, a floor in an office building, or a city block. This type of localization can be proximity-based, such as a node is located in Region A if an anchor in Region A detects it there. Another technique to localize using hop count 58. Because the approximate transmission range of the node radio is known, observing the number of message hops to a set of anchor nodes will constrain the target node to a specific region.

\subsection{Localization Phase}

The signal data obtained the measurement phase can be used to determine the approximate position of the target node. Common localization techniques for MWSNs are based on ranging, whereby distance or angle approximations are obtained. Because range data are often corrupted by noisy signal measurements, optimization methods are employed to filter the noise and arrive at a more accurate position estimate. 
(a)

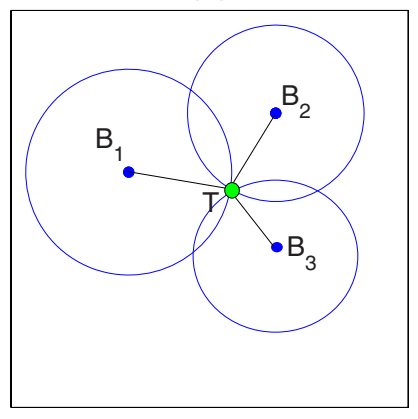

(b)

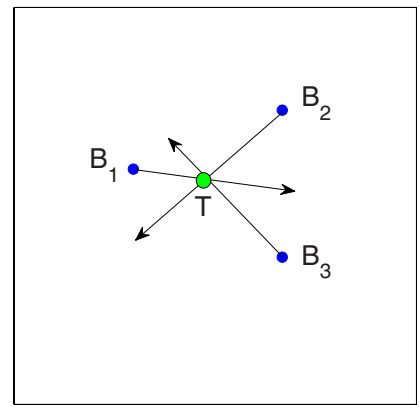

Fig. 5. The position of a target node $(T)$ is estimated based on the known positions of beacons $\left(B_{i}\right)$ using (a) lateration or (b) angulation

Lateration. When ranges between landmarks and the mobile node can be determined, lateration is used to estimate position [59]. Figure [5a illustrates the method. For two-dimensional localization, three range measurements from known positions are required. Each range can be represented as the radius of a circle, with the anchor node situated at the center. Without measurement noise, the three circles would intersect at exactly one point, the location of the target node. However, in the presence of noise, the three circles will overlap, and the target node will likely (but not necessarily) be contained within that region.

Angulation. When anchor bearings or angular separation between anchors and the mobile node can be obtained, angulation can be used to determine the position of the mobile node [60, [61, [46], 62]. This is pictured in Figure 5b. For tri-angulation, when two anchors are used, the target position will be identified as the third point in a triangle of two known angles (the bearings from each anchor), and the length of one side (the distance between anchor nodes). Often more than two anchor bearings are used, and target position is determined by the intersection of all bearings, as illustrated in the figure. In the presence of measurement noise, the bearings will not all intersect at the exact same point, but will instead define a region where the target node is likely to be.

Cellular Proximity. An alternative approach is the range-free method (39, [63, [58]), whereby a node is localized to the region in which it is detected. This method generally provides a more course-grained position estimate, and depends on the density of infrastructure nodes.

Dead Reckoning. A widely used localization technique for mobile robots is dead reckoning [3, 64, 65], 7]. Robots obtain their current velocity from wheel encoders or other means, and use this information in conjunction with the amount of time that has elapsed since the last update to derive current position and heading. The major drawback of this approach is that the position 
estimation accrues error over time, primarily because of noisy encoder data due to uneven surfaces, wheel slippage, dust, and other factors.

Estimation Methods. When measurement data is noisy, or the system is underdefined, state estimation methods can be used. There exist a number of estimation methods, but the two main approaches are: (1) maximum likelihood estimation (MLE) 66, which estimates the values of the state based on measured data only, and no prior information about the state is used, and (2) sequential Bayesian estimation (SBE) 67], which estimates state values based on measurements, as well as prior information.

MLE methods such as 68 and 69 find the estimates for the system state by maximizing the likelihood of the measured data. In other words, MLE picks the values of the system parameters that make the observed data "more likely" than any other values for the parameters. The data likelihood is computed using a measurement model that relates the measured data to the system state.

In SBE, the system state is iteratively estimated using the recursive Bayes rule which states that the posterior is proportional to the product of the data likelihood and the predicted prior. Such methods are used in [55], [53], and [70]. Like MLE, the data likelihood is computed using a measurement model. The solution to SBE is generally intractable and cannot be determined analytically. Optimal solutions do exist in a restrictive set of cases, such as the Kalman Filter (KF) [71] and gridbased filters. More general suboptimal solutions exist, such as Extended Kalman Filter (EKF) 72 and Particle Filters (PF) that approximate the optimal Bayesian estimation. The sequential Monte Carlo (SMC) 64 method is a PF that provides a suboptimal solution by approximating the posterior density by a set of random samples (also called particles) with associated weights. As the number of particles becomes very large, the particle filter approaches an optimal solution.

\subsection{The Effect of Mobility on Localization}

Typically, localization of mobile sensors is performed in order to track them, or for navigational purposes. However, when sensors are mobile, we encounter additional challenges and must develop methods to address them.

One of these challenges is localization latency. If the time to perform the localization takes too long, the sensor will have significantly changed its position since the measurement took place. For example, robot navigation requires periodic position estimates in order to derive the proper control outputs for wheel angular velocity. If the robot is traveling at $1 \mathrm{~m} / \mathrm{s}$ and the localization algorithm takes 5 seconds to complete from the time the ranging measurements were taken, the robot might be 5 meters off from its intended position.

Mobility may also impact the localization signal itself. For example, the frequency of the signal may undergo a Doppler shift, introducing error into the measurement. Doppler shifts occur when the transmitter of a signal is moving relative to the receiver, as illustrated in Figure 6. The resulting shift in frequency is related to the positions and relative speed of the two nodes. mTrack 32 takes this 
(a)

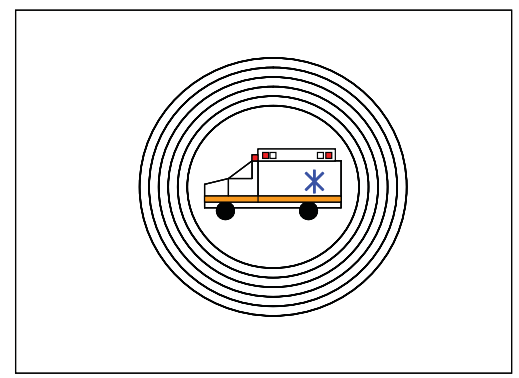

(b)

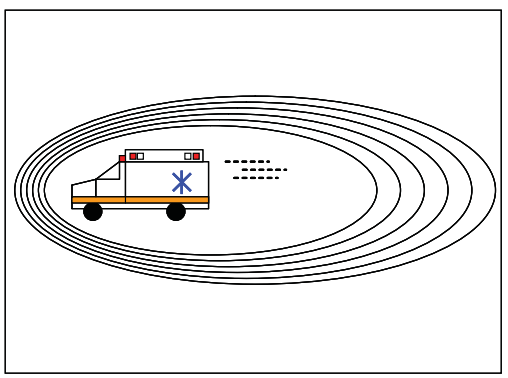

Fig. 6. (a) The frequency of a signal does not change when the transmitter and receiver are moving at the same relative speed. (b) However, when the transmitter and receiver are moving relative to one another, the signal will undergo a Doppler shift.

Doppler effect into account and uses it to refine its position estimate. Other approaches [9], 6] use the Doppler effect to directly solve for position and velocity.

If the localization technique requires line of sight (LOS), there is the possibility that the mobile sensor will move from a position with good LOS, to a position with poor LOS. When this is the case, a dense network of nodes is required to ensure there is always LOS to the mobile node, wherever it may move.

\subsection{Centralized vs. Distributed Algorithms}

The resource constraints inherent in WSNs pose a challenge when it comes to executing certain localization algorithms, because they require extensive memory and processor bandwidth, especially when dealing with a large number of sensors, or when using complex statistical methods to estimate range or position [25]. A centralized localization algorithm runs on a base station, and all participating nodes must forward their measurement data to the base station. The advantage of the centralized approach is an algorithm can be designed that has more accuracy, precision, and can process greater amounts of data. On the other hand, base station processing suffers from the common pitfalls of centralization, such as poor scalability, single point of failure, data routing complexity, and greater power consumption (especially for nodes closer to the base station).

When nodes are mobile, the decision to use centralized or distributed processing becomes even more important. Mobility requires continuous and rapid localization. Although centralized localization techniques exist for mobile sensors [32, 9], they are usually not fast enough for certain applications, such as navigation. For example, mTrack 32 reports a latency of approximately 5 seconds. dNav [6], on the other hand, is distributed, and takes less than 1 second on average to return position and velocity estimates.

\subsection{The Impact of Environment on Localization}

The environment plays a significant role in the effectiveness of a localization method. As a result, there is no one localization method that will be accurate 
for all situations. Different environmental factors are listed below, as well as the effect they have on the aforementioned localization methods.

Ambient temperature, pressure, and humidity can affect localization accuracy, because these directly impact the crystal oscillator in the transceiver. Furthermore, it has been well established that radio wave propagation is affected by precipitation, including moisture in the air, therefore localization techniques that use RF measurements can be impaired under these conditions [73]. One of the biggest problems with GPS is that it does not work reliably under water, indoors, or even when it is cloudy. This is because the GPS receiver requires line of sight to up to four satellites orbiting the planet [4].

At present there is a major effort underway to develop accurate localization methods in indoor environments. Indoor applications that require node position estimation are challenging because most propagation methods and measurement techniques suffer from multipath effects [74, where obstacles (e.g. walls, furniture, people, etc.) cause signal reflections that interfere with each other. In addition, many of the existing localization techniques that provide good accuracy outdoors, will not work indoors.

\section{MWSN Applications with Localization Requirements}

Although MWSNs are still in their infancy, several types of applications have already been developed in which localization plays an integral part. The applications fall under four main categories, (a) commercial, (b) environmental, (c) civil, and (d) military, however, most span more than just one of these.

\subsection{Commercial}

As MWSNs grow in popularity, we expect to see a burst of applications in the commercial sector that require some kind of position data.

- Service Industry. One such area is the service industry. Companies such as Skilligent 75] are developing software protocols for service robots that perform tasks such as basic patient care in nursing homes, maintenance and security in office buildings, and food and concierge service in restaurants and hotels. All of these applications require a mechanism for position estimation. Skilligent uses a visual localization system based on pattern matching. Objects are used as landmarks, and are loaded into the system a priori, or dynamically at runtime. The robot learns its position by matching video images with landmark information.

- Housekeeping. The iRobot Roomba [76] is an automated vacuum cleaning robot for domestic use. The Roomba creates a map of the room as it moves by using feedback from a variety of bumper and optical sensors. Wheel encoders provide run-time position information that enable it to cover the entire room. The Roomba also uses a self-docking station to automatically recharge its batteries. 


\subsection{Environmental}

MWSNs have become a valuable asset for environmental monitoring. This is thanks in part to their ability to be deployed in remote areas and for their ability to gather data of wide areas of interest.

- Wildlife Tracking. ZebraNet [8] is an early MWSN, in which mote-scale wireless devices were fitted to zebras for the purpose of tracking their movement. Due to the remote region, there was no cellphone coverage, so data was routed through the peer-to-peer network to mobile base stations. The zebras were not constrained to certain areas, and other than the small devices attached to their bodies, left undisturbed. To accomplish this level of tracking without the use of MWSNs would not be possible.

- Pollution Monitoring. A mobile air quality monitoring system is presented in 77. Sensor nodes that measure specific pollutants in the air are mounted on vehicles. As the vehicles move along the roadways, the sensors sample the air, and record the concentration of various pollutants along with location and time. When the sensors are in the proximity of access points, the data are uploaded to a server and published on the web.

\subsection{Civil}

One of the areas that has great potential for MWSN utility is that of civil services. This includes those non-military municipal applications that keep society running efficiently and safely.

- Pothole Detection. In 78, a system is developed to detect potholes on city streets. Deployed on taxi cabs, the sensor nodes contain an accelerometer, and can communicate using either opportunistic WiFi or cellular networks.

- Wireless E-911. In North America, the Enhanced 911 emergency telecommunications service, or E911 79, was established to connect callers with emergency services in a manner that would associate a physical location with the phone number of the caller. Wireless E-911 is the second phase of the E911 service mandated by the FCC, which requires wireless cellular devices to automatically provide user location when the service is invoked. This is an important requirement, however, its implementation is non-trivial, and different carriers choose to use different methods, including embedded GPS chips, and multilateration and angulation based on the known locations of cell towers.

\subsection{Military / Aerospace}

One of the biggest promoters, as well as one of the biggest funders, of wireless sensor technology is the military. There is a clear interest in localization services, tracking friendly and hostile entities, and navigation of autonomous robots, and intensive research is carried out in this area.

- Shooter Detection / Weapon Classification. In [80], a soldier-wearable sensor system is developed that not only identifies the location of an enemy 
sniper, but also identifies the weapon being fired. Each sensor consists of an array of microphones mounted on the helmet of a soldier. The sensor observes both the shock wave of the projectile, as well as the muzzle blast from the weapon, and based on TDOA, as well as properties of the acoustic signal, is able to triangulate the enemy position and classify the weapon type.

- Autonomous Deployment. In 81] an unattended aerial vehicle is used for sensor network deployment and repair. Such deployments aid the military in battlefield surveillance and command and control field operations.

\section{Conclusion}

In this paper, we presented a survey and taxonomy on localization for mobile wireless sensor networks. Localization in MWSNs entails new challenges that result from integrating resource-constrained wireless sensors on a mobile platform. The localization methods and algorithms that provide greater accuracy on larger-footprint mobile entities with fewer resource restrictions are no longer applicable. Similarly, centralized and high-latency localization techniques for static WSNs are undesirable for the majority of MWSN applications.

There are several directions for future work in MWSN localization. Reducing localization latency is one of the most important benchmarks for MWSNs. Currently, a tradeoff exists between the rapid execution of an algorithm and its accuracy. Additional work is needed that focused on reducing run-time latency, while maintaining positioning accuracy. In addition, the majority of localization algorithms to date are centralized. For mobile sensor localization, this is often a poor design choice, due to the additional latency and energy costs incurred. The development of more distributed localization techniques would be a welcome addition to MWSN localization. There is much interest in localization in urban and indoor areas where obstacles such as vehicles, walls, people, and furniture cause multipath propagation and loss of line of sight. Most current methods use some variation of RSS profiling, in conjunction with optimization techniques. However, new methods are required as we expand mobile sensing to areas where training data cannot safely be obtained, such as urban war zones or burning buildings. Lastly, mobile actuated sensors are now being developed with motesized form factors. Like embedded sensor nodes, these devices also have resource constraints, which limit their ability to navigate a sensing region in the same way a robot with a full array of sensors and powerful processing capability might. We can expect to see many advances in mobile sensor navigation in the near future.

Acknowledgements. This work was supported in part by ARO MURI grant W911NF-06-1-0076, NSF grant CNS-0721604, and NSF CAREER award CNS0347440. The authors also wish to thank Manish Kushwaha for his valuable input. 


\section{References}

1. Ekici, E., Gu, Y., Bozdag, D.: Mobility-based communication in wireless sensor networks. Communications Magazine, IEEE 44(7), 56-62 (2006)

2. Munir, S.A., Ren, B., Jiao, W., Wang, B., Xie, D., Ma, J.: Mobile wireless sensor network: Architecture and enabling technologies for ubiquitous computing. In: Proceedings of the 21st International Conference on Advanced Information Networking and Applications Workshops, AINAW (2007)

3. Tilak, S., Kolar, V., Abu-Ghazaleh, N.B., Kang, K.D.: Dynamic localization control for mobile sensor networks. In: Proceedings of the IEEE International Workshop on Strategies for Energy Efficiency in Ad Hoc and Sensor Networks (2005)

4. Hofmann-Wellenhof, B., Lichtenegger, H., Collins, J.: Global Positioning System: Theory and Practice, 4th edn. Springer, Heidelberg (1997)

5. Munir, S.A., Ren, B., Jiao, W., Wang, B., Xie, D., Ma, J.: Mobile wireless sensor network: Architecture and enabling technologies for ubiquitous computing. In: International Conference on Advanced Information Networking and Applications Workshops, vol. 2, pp. 113-120 (2007)

6. Amundson, I., Koutsoukos, X., Sallai, J.: Mobile sensor localization and navigation using RF doppler shifts. In: 1st ACM International Workshop on Mobile Entity Localization and Tracking in GPS-less Environments, MELT (2008)

7. Fang, L., Antsaklis, P.J., Montestruque, L., Mcmickell, M.B., Lemmon, M., Sun, Y., Fang, H., Koutroulis, I., Haenggi, M., Xie, M., Xie, X.: Design of a wireless assisted pedestrian dead reckoning system - the NavMote experience. In: IEEE Transactions on Instrumentation and Measurement, vol. 54(6), pp. 2342-2358 (2005)

8. Juang, P., Oki, H., Wang, Y., Martonosi, M., Peh, L., Rubenstein, D.: Energyefficient computing for wildlife tracking: Design tradeoffs and early experiences with zebranet. In: Proc. of ASPLOS-X (2002)

9. Kusý, B., Lédeczi, A., Koutsoukos, X.: Tracking mobile nodes using RF doppler shifts. In: SenSys 2007: Proceedings of the 5th international conference on Embedded networked sensor systems, pp. 29-42. ACM, New York (2007)

10. Crossbow MICAz (MPR2400) Radio Module, http://www.xbow.com/Products/productsdetails.aspx?sid=101

11. Dutta, P., Grimmer, M., Arora, A., Bibyk, S., Culler, D.: Design of a wireless sensor network platform for detecting rare, random, and ephemeral events. In: Proc. of IPSN/SPOTS (April 2005)

12. Polastre, J., Szewczyk, R., Culler, D.: Telos: Enabling ultra-low power wireless research. In: Proc. of IPSN/SPOTS (April 2005)

13. Dantu, K., Rahimi, M., Shah, H., Babel, S., Dhariwal, A., Sukhatme, G.S.: Robomote: enabling mobility in sensor networks. In: The Fourth International Symposium on Information Processing in Sensor Networks, IPSN (2005)

14. Friedman, J., Lee, D.C., Tsigkogiannis, I., Wong, S., Chao, D., Levin, D., Kaisera, W.J., Srivastava, M.B.: Ragobot: A new platform for wireless mobile sensor networks. In: International Conference on Distributed Computing in Sensor Systems, DCOSS (2005)

15. Bergbreiter, S., Pister, K.S.J.: CotsBots: An off-the-shelf platform for distributed robotics. In: Proceedings of the IEEE/RSJ International Conference on Intelligent Robots and Systems, IROS (2003)

16. Shah, R., Roy, S., Jain, S., Brunette, W.: Data mules: modeling a three-tier architecture for sparse sensor networks. In: Proceedings of the First IEEE International Workshop on Sensor Network Protocols and Applications (2003) 
17. Wang, G., Cao, G., Porta, T., Zhang, W.: Sensor relocation in mobile sensor networks. In: IEEE INFOCOM (2005)

18. Liu, B., Brass, P., Dousse, O., Nain, P., Towsley, D.: Mobility improves coverage of sensor networks. In: Proceedings of the 6th ACM international symposium on Mobile ad hoc networking and computing (MobiHoc), pp. 300-308 (2005)

19. Gandham, S., Dawande, M., Prakash, R., Venkatesan, S.: Energy efficient schemes for wireless sensor networks with multiple mobile base stations. In: IEEE Global Telecommunications Conference, GLOBECOM (2003)

20. Kansal, A., Somasundara, A.A., Jea, D.D., Srivastava, M.B., Estrin, D.: Intelligent fluid infrastructure for embedded networks. In: Proceedings of the 2nd international conference on Mobile systems, applications, and services (MobiSys), pp. 111-124 (2004)

21. Al-Karaki, J.N., Kamal, A.E.: Routing techniques in wireless sensor networks: a survey. IEEE Wireless Communications 11(6), 6-28 (2004)

22. Abolhasan, M., Wysocki, T., Dutkiewicz, E.: A review of routing protocols for mobile ad hoc networks. Ad Hoc Networks 2(1), 1-22 (2004)

23. Wang, Q., Hempstead, M., Yang, W.: A realistic power consumption model for wireless sensor network devices. In: 3rd Annual IEEE Communications Society on Sensor and Ad Hoc Communications and Networks (SECON), vol. 1, pp. 286-295 (2006)

24. Hightower, J., Borriello, G.: Location systems for ubiquitous computing. IEEE Computer 34(8), 57-66 (2001)

25. Mao, G., Fidan, B., Anderson, B.D.O.: Wireless sensor network localization techniques. Computer Networks 51(10), 2529-2553 (2007)

26. Brooks, R.R., Griffin, C., Friedlander, D.S.: Self-organized distributed sensor network entity tracking. The International Journal of High Performance Computing Applications 16(3) (2002)

27. Moore, D., Leonard, J., Rus, D., Teller, S.: Robust distributed network localization with noisy range measurements. In: SenSys 2004: Proceedings of the 2nd international conference on Embedded networked sensor systems, pp. 50-61 (2004)

28. Girod, L., Lukac, M., Trifa, V., Estrin, D.: The design and implementation of a self-calibrating acoustic sensing platform. In: Proc. of ACM SenSys (November 2006)

29. Elson, J., Girod, L., Estrin, D.: Fine-grained network time synchronization using reference broadcasts. SIGOPS Oper. Syst. Rev. 36(SI), 147-163 (2002)

30. Kusý, B., Dutta, P., Levis, P., Maróti, M., Lédeczi, A., Culler, D.: Elapsed time on arrival: a simple and versatile primitive for canonical time synchronization services. International Journal of Ad Hoc and Ubiquitous Computing 2(1) (2006)

31. Maróti, M., Kusý, B., Balogh, G., Völgyesi, P., Nádas, A., Molnár, K., Dóra, S., Lédeczi, A.: Radio interferometric geolocation. In: Proc. of ACM SenSys (November 2005)

32. Kusý, B., Sallai, J., Balogh, G., Lédeczi, A., Protopopescu, V., Tolliver, J., DeNap, F., Parang, M.: Radio interferometric tracking of mobile wireless nodes. In: Proc. of MobiSys (2007)

33. Harter, A., Hopper, A., Stegglesand, P., Ward, A., Webster, P.: The anatomy of a context-aware application. In: Mobile Computing and Networking, pp. 59-68 (1999)

34. Priyantha, N.B., Chakraborty, A., Balakrishnan, H.: The Cricket location-support system. In: Proc. of MobiCom (August 2000)

35. McCarthy, M., Duff, P., Muller, H.L., Randell, C.: Accessible ultrasonic positioning. IEEE Pervasive Computing 5(4), 86-93 (2006) 
36. Chen, J., Yao, K., Hudson, R.: Source localization and beamforming. Signal Processing Magazine, IEEE 19(2), 30-39 (2002)

37. Lédeczi, A., Nádas, A., Völgyesi, P., Balogh, G., Kusý, B., Sallai, J., Pap, G., Dóra, S., Molnár, K., Maróti, M., Simon, G.: Countersniper system for urban warfare. ACM Transactions on Sensor Networks 1(1), 153-177 (2005)

38. Williams, S.M., Frampton, K.D., Amundson, I., Schmidt, P.L.: Decentralized acoustic source localization in a distributed sensor network. Applied Acoustics $67(2006)$

39. Want, R., Hopper, A., Falcao, V., Gibbons, J.: The active badge location system. ACM Transactions on Information Systems 40 (1992)

40. Brassart, E., Pegard, C., Mouaddib, M.: Localization using infrared beacons. Robotica 18(2), 153-161 (2000)

41. Kemper, J., Linde, H.: Challenges of passive infrared indoor localization. In: Proceedings of 5th Workshop on Positioning, Navigation and Communication (WPNC), pp. 63-70 (2008)

42. Kusý, B., Balogh, G., Völgyesi, P., Sallai, J., Nádas, A., Lédeczi, A., Maróti, M., Meertens, L.: Node-density independent localization. In: Proc. of IPSN/SPOTS (April 2006)

43. Römer, K.: The lighthouse location system for smart dust. In: Proceedings of the 1st International Conference on Mobile Systems, Applications and Services (MobiSys), pp. 15-30 (2003)

44. Stoleru, R., He, T., Stankovic, J.A., Luebke, D.: A high-accuracy, low-cost localization system for wireless sensor networks. In: Proceedings of ACM SenSys (November 2005)

45. Bekris, K.E., Argyros, A.A., Kavraki, L.E.: Angle-based methods for mobile robot navigation: Reaching the entire plane. In: International Conference on Robotics and Automation (2004)

46. Niculescu, D., Nath, B.: Ad hoc positioning system (APS) using AOA. In: Proceedings of the Twenty-Second Annual Joint Conference of the IEEE Computer and Communications Societies, INFOCOM (2003)

47. Friedman, J., Charbiwala, Z., Schmid, T., Cho, Y., Srivastava, M.: Angle-of-arrival assisted radio interferometry (ARI) target localization (2008)

48. Priyantha, N.B., Balakrishnan, H., Demaine, E.D., Teller, S.: Mobile-assisted localization in wireless sensor networks. In: Proceedings of the IEEE 24th Annual Joint Conference of the IEEE Computer and Communications Societies (INFOCOM), vol. 1 (2005)

49. Caffery Jr., J.J.: A new approach to the geometry of toa location. In: 52nd Vehicular Technology Conference, 2000. IEEE VTS-Fall VTC 2000, vol. 4, pp. 1943-1949 (2000)

50. Wang, X., Wang, Z., O'Dea, B.: A toa-based location algorithm reducing the errors due to non-line-of-sight (nlos) propagation. IEEE Transactions on Vehicular Technology 52(1), 112-116 (2003)

51. Günther, A., Hoene, C.: Measuring round trip times to determine the distance between WLAN nodes. In: Boutaba, R., Almeroth, K.C., Puigjaner, R., Shen, S., Black, J.P. (eds.) NETWORKING 2005. LNCS, vol. 3462, pp. 768-779. Springer, Heidelberg (2005)

52. Lee, H., Wicke, M., Kusy, B., Guibas, L.: Localization of mobile users using trajectory matching. In: MELT 2008: Proceedings of the first ACM international workshop on Mobile entity localization and tracking in GPS-less environments, pp. 123-128 (2008) 
53. Madigan, D., Einahrawy, E., Martin, R., Ju, W.H., Krishnan, P., Krishnakumar, A.: Bayesian indoor positioning systems. In: INFOCOM 2005. Proceedings of IEEE 24th Annual Joint Conference of the IEEE Computer and Communications Societies, March 2005, vol. 2, pp. 1217-1227 (2005)

54. Bahl, P., Padmanabhan, V.N.: Radar: An in-building RF-based user-location and tracking system. In: Proc. IEEE INFOCOM, March 2000, vol. 2, pp. 775-784 (2000)

55. Ladd, A., Bekris, K., Rudys, A., Wallach, D., Kavraki, L.: On the feasibility of using wireless ethernet for indoor localization. IEEE Transactions on Robotics and Automation 20(3), 555-559 (2004)

56. Ledeczi, A., Volgyesi, P., Sallai, J., Thibodeaux, R.: A novel RF ranging method. In: Sixth Workshop on Intelligent Solutions in Embedded Systems, WISES (2008)

57. Chang, H.I., Tian, J.B., Lai, T.T., Chu, H.H., Huang, P.: Spinning beacons for precise indoor localization. In: Proceedings of the Sixth ACM conference on Embedded network sensor systems, SenSys (2008)

58. Niculescu, D., Nath, B.: Dv based positioning in ad hoc networks. Journal of Telecommunication Systems 22, 267-280 (2003)

59. Manolakis, D.: Efficient solution and performance analysis of 3-D position estimation by trilateration. IEEE Transactions on Aerospace and Electronic Systems 32(4), 1239-1248 (1996)

60. Esteves, J., Carvalho, A., Couto, C.: Generalized geometric triangulation algorithm for mobile robot absolute self-localization (2003)

61. McGillem, C., Rappaport, T.: A beacon navigation method for autonomous vehicles. IEEE Transactions on Vehicular Technology 38(3), 132-139 (1989)

62. Betke, M., Gurvits, L.: Mobile robot localization using landmarks 13(2), 251-263 (April 1997)

63. Shang, Y., Ruml, W., Zhang, Y., Fromherz, M.: Localization from connectivity in sensor networks. IEEE Transactions on Parallel and Distributed Systems 15(11), 961-974 (2004)

64. Hu, L., Evans, D.: Localization for mobile sensor networks. In: Proceedings of the 10th annual international conference on Mobile computing and networking, MobiCom (2004)

65. Zhang, P., Martonosi, M.: Locale: Collaborative localization estimation for sparse mobile sensor networks. In: Proceedings of the 7th international conference on Information processing in sensor networks, IPSN (2008)

66. Kay, S.M.: Fundamentals of Statistical Signal Processing, Volume I: Estimation Theory. Prentice-Hall, Englewood Cliffs (1993)

67. Arulampalam, M., Maskell, S., Gordon, N., Clapp, T.: A tutorial on particle filters for online nonlinear/non-gaussian bayesian tracking. IEEE Transactions on Signal Processing 50(2), 174-188 (2002)

68. Kuang, X., Shao, H.: Maximum likelihood localization algorithm using wireless sensor networks. In: First International Conference on Innovative Computing, Information and Control (ICICIC), vol. 3, pp. 263-266 (2006)

69. Mendalka, M., Kulas, L., Nyka, K.: Localization in wireless sensor networks based on zigbee platform. In: 17th International Conference on Microwaves, Radar and Wireless Communications (MIKON), pp. 1-4 (2008)

70. Fox, V., Hightower, J., Liao, L., Schulz, D., Borriello, G.: Bayesian filtering for location estimation. Pervasive Computing, IEEE 2(3), 24-33 (2003)

71. Kalman, R.E.: A new approach to linear filtering and prediction problems. Trans. ASME, Journal of Basic Engineering (1960) 
72. Welch, G., Bishop, G.: An introduction to the Kalman filter. Technical Report TR 95-041, Department of Computer Science, University of North Carolina at Chapel Hill (2004)

73. Sizun, H.: Radio wave propagation for telecommunication applications. Springer, Heidelberg (2004)

74. Hashemi, H.: The indoor radio propagation channel. Proceedings of the IEEE 81(7), 943-968 (1993)

75. Skilligent: Skilligent visual localization system, http://www.skilligent.com/products/robot-navigation.shtml

76. iRobot: Roomba vacuum cleaning robot, http://www.irobot.com

77. Völgyesi, P., Nádas, A., Koutsoukos, X., Lédeczi, A.: Air quality monitoring with sensormap. In: Proceedings of the 7th international conference on Information processing in sensor networks (IPSN), pp. 529-530 (2008)

78. Eriksson, J., Girod, L., Hull, B., Newton, R., Madden, S., Balakrishnan, H.: The pothole patrol: using a mobile sensor network for road surface monitoring. In: Proceedings of the 6th international conference on Mobile systems, applications, and services, MobiSys (2008)

79. Federal Communications Commission: Enhanced 911, http://www.fcc.gov/pshs/services/911-services/enhanced911/

80. Völgyesi, P., Balogh, G., Nádas, A., Nash, C., Lédeczi, A.: Shooter localization and weapon classification with soldier-wearable networked sensors. 5th International Conference on Mobile Systems, Applications, and Services, MobiSys (2007)

81. Corke, P., Hrabar, S., Peterson, R., Rus, D., Saripalli, S., Sukhatme, G.: Autonomous deployment and repair of a sensor network using an unmanned aerial vehicle. In: IEEE International Conference on Robotics and Automation, pp. 3602-3609 (2004) 